\title{
A NOTE ON THE STEADY STATE SOLUTIONS OF THE HEAT EQUATION ${ }^{1}$
}

W. FULKS

1. Introduction. Let $R$ be a bounded region (open connected set) in $E_{n}\left[x=\left(x_{1}, x_{2}, \cdots, x_{n}\right)\right]$, and $B$ be its boundary. We shall say that $R$ is a regular region (Tychonoff [8]: fundamental domain) for the heat equation

$$
\Delta u=u_{t}
$$

if there exists a unique solution to the Dirichlet problem for continuous data. By this we mean that for each

$$
\phi(x) \in C \text { (i.e. is continuous), } \quad x \in \bar{R}=R \cup B,
$$

and

$$
\psi(\xi, t) \in C, \quad \xi \in B, t \geqq 0,
$$

with

$$
\phi(\xi)=\psi(\xi, 0), \quad \xi \in B,
$$

there exists a unique function

$$
u(x, t) \in C, \quad x \in \bar{R}, t \geqq 0
$$

for which

$$
\begin{aligned}
\Delta u & =u_{t}, & & x \in R, t>0, \\
u(x, 0) & =\phi(x), & & x \in \bar{R}, \\
u(\xi, t) & =\psi(\xi, t), & & \xi \in B, t \geqq 0 .
\end{aligned}
$$

Similarly we will say that $R$ is regular for Laplace's equation

$$
\Delta u=0
$$

if for each $\psi(\xi) \in C, \xi \in B$, there exists a unique $u(x) \in C$ for $x \in \bar{R}$, such that $\Delta u=0$ for $x \in R$ and $u(\xi)=\psi(\xi), \xi \in B$.

Tychonoff [8] has shown that if $R$ is regular for the heat equation it is regular for Laplace's equation by showing that under suitable conditions the solution of the heat equation approaches a steady

Presented to the Society, August 31, 1954; received by the editors August 24, 1955.

${ }^{1}$ The work on this paper was sponsored by the ONR Contract number Nonr386(00) (NR044004). 
state, which gives the desired solution to the Dirichlet problem for $\Delta u=0$. Similar problems have been treated by Rosenbloom and Milgram $[4 ; 5]$ for Riemannian manifolds, and Rosenbloom [7] has given a different solution to the original problem of Tychonoff. We give here another proof, and also show the pointwise monotone character of the convergence under reasonable conditions.

2. Regularity proof. We take as our point of departure another theorem of Tychonoff [8], which shows that if $R$ is regular for $\Delta u=u_{t}$, the condition $\phi(\xi)=\psi(\xi, 0)$ may be replaced by a requirement that $u(x, t)$ remain bounded, without jeopardizing the existence and uniqueness of the solution, which, however, will not in general be continuous for $x \in B, t=0$.

We shall have need of a classical minimum (and maximum) principle: if $u(x, t)$ be a solution of (1) for $x \in R, t>0$, and lim inf $u(x, t)$ $\geqq 0$ as $(x, t) \rightarrow\left(x^{0}, 0\right), x^{0} \in \bar{R}$, and as $(x, t) \rightarrow\left(\xi^{0}, t_{0}\right), \xi^{0} \in B, t_{0} \geqq 0$, then $u(x, t) \geqq 0$ for $x \in R, t>0$. (For a proof in the one-dimensional case, which is not essentially different in any number of dimensions, see Widder [9].) If we consider $-u(x, t)$ our minimum principle becomes a maximum principle.

To show that $R$ is regular for $\Delta u=0$ it suffices to show that each point $\xi^{0} \in B$ is a regular point, and for this it suffices to show that at $\xi^{0}$ we can construct a barrier. (See Kellogg [3].)

To this end let $\xi^{0}$ be an arbitrary point of $B$, let $D$ be the diameter of $R$, and let $r=r_{\xi^{0}}=\left\|\xi^{0}-\xi\right\|$ be distance from $\xi^{0}$ to a variable point $\xi$ of $B$. Let $u(x, t)$ be the solution of the problem

$$
\begin{array}{rlrl}
\Delta u & =u_{t}, & & x \in R, t>0, \\
u(x, 0) & =D, & & x \in \bar{R}, \\
u(\xi, t) & =\left\{\begin{array}{ll}
(1-t) D+t \cdot r, & 0 \leqq t \leqq 1 \\
r, & t \geqq 1
\end{array}\right\}, & \xi \in B .
\end{array}
$$

By our minimum and maximum principles

$$
0 \leqq u(x, t) \leqq D \text {, }
$$

and

$$
u\left(\xi^{0}, t\right)=0, \quad t \geqq 1 .
$$

Choose $t_{0}>1$, and consider $u\left(x, t_{0}\right)$ as a function of $x$ in $\bar{R}$. We shall show $u\left(x, t_{0}\right)>0$ except at $x=\xi^{0}$. For it cannot vanish for $x=\xi \neq \xi^{0}$, $\xi \in B$, for $u\left(\xi, t_{0}\right)=r>0$, and if $u\left(x, t_{0}\right)=0$, for some $x \in R$, then $u(x, t) \equiv 0 x \in R, 0 \leqq t \leqq t_{0}$ (see Nirenberg [6]). But this contradicts our assumption that $u(x, 0)=D>0$. Hence $u\left(x, t_{0}\right)$ vanishes only at $\xi^{0}$ 
and consequently outside any sphere with center $\xi^{0}$ it has a positive lower bound.

Consider now, for $h>0$,

$$
v(x, t)=u(x+t, h)-u(x, t) .
$$

This function is a solution of $\Delta u=u_{t}$ for $x \in R, t>0$, and

$$
\begin{aligned}
v(x, 0) & =u(x, h)-u(x, 0)=u(x, h)-D \leqq 0 . \\
v(\xi, t) & =u(\xi, t+h)-u(\xi, t) \leqq 0,
\end{aligned}
$$

so that by our maximum principle

$$
v(x, t) \leqq 0, \quad x \in R, \quad t>0 .
$$

Hence $\partial u / \partial t \leqq 0$. But at $t_{0}$

$$
\Delta u\left(x, t_{0}\right)=\left.\frac{\partial}{\partial t} u(x, t)\right|_{t_{0}} \leqq 0 .
$$

Hence we have produced a super-harmonic function with the requisite properties to serve as a barrier. Since $\xi^{0}$ was an arbitrary point of $B$ this completes the proof that $R$ is regular for $\Delta u=0$.

3. Convergence to the steady state. We next consider convergence to the steady state. Let $\psi(\xi, t) \geqq 0$ be continuous for $\xi \in B, t \geqq 0$ and nondecreasing in $t$, and let it converge to a continuous function $\psi(\xi)$ as $t$ tends toward infinity. Let $u(x, t)$ be the bounded solution to the problem

$$
\begin{aligned}
& \Delta u=u_{t}, \quad x \in R, \quad t>0, \\
& u(x, 0)=0, \quad x \in R, \\
& u(\xi, t)=\psi(\xi, t), \quad \xi \in B, \quad t>0 .
\end{aligned}
$$

We shall show that $u(x, t)$ is positive and is monotone nondecreasing in $t$ and converges uniformly to the solution of

$$
\begin{aligned}
\Delta u & =0, & & x \in R \\
u(\xi) & =\psi(\xi), & & \xi \in B .
\end{aligned}
$$

For this purpose we need another result of Tychonoff [8], namely, if $\phi(x)$ is continuous in $\bar{R}$, and $G_{R}(x, y, t)$ is the Green's function for $R$, then

$$
\pi(x, t)=\int_{R} G_{R}(x, y, t) \phi(y) d y \quad\left[d y=d y_{1} \cdots d y_{n}\right]
$$

is the solution of the problem 


$$
\begin{aligned}
& \Delta \pi=\pi_{t}, \quad x \in R, \quad t>0, \\
& \pi(x, 0)=\phi(x), \quad x \in R, \\
& \pi(\xi, t)=0, \quad \xi \in B, \quad t>0 \text {. }
\end{aligned}
$$

Since $G \geqq 0$ it is clear that if $\phi \geqq 0$, so is $\pi$. Though Tychonoff does not point it out, it is easy to see that for any given $\xi \in B$

$$
0 \leqq \liminf _{(x, t) \rightarrow(\xi, 0)} \pi(x, t) \leqq \limsup _{(x, t) \rightarrow(\xi, 0)} \pi(x, t) \leqq \phi(\xi) .
$$

Let us now choose $\phi(x)$ to be any continuous positive extension of $\psi(\xi, 0)$ into $\bar{R}$, and let $w(x, t)$ be the solution to the problem

$$
\begin{aligned}
& \Delta w=w_{t}, \quad x \in R, \quad t>0, \\
& w(x, 0)=\phi(x), \quad x \in \bar{R}, \\
& w(\xi, t)=\psi(\xi, t), \quad \xi \in R, \quad t \geqq 0 .
\end{aligned}
$$

Consider $w-\pi$ : this function is a solution of the heat equation and attains 0 and $\psi(\xi, t)$ as initial and boundary values, respectively. Hence this must be $u(x, t)$. Now lim inf $u(x, t) \geqq 0$ for all boundary and initial points with the possible exception of those of the form $(\xi, 0)$ where $\xi \in B$. But as $(x, t) \rightarrow(\xi, 0)$

$\lim \inf u(x, t) \geqq \lim \inf w(x, t)-\lim \sup \pi(x, t)=\psi(\xi, 0)-\phi(\xi)=0$.

So that $u(x, t)$ is clearly positive.

Next we wish to show that the auxiliary function

$$
v(x, t)=u(x, t+h)-u(x, t),
$$

where $h$ is fixed and positive, is itself positive. To see this let $\phi(x)$ be any continuous extension of $\psi(\xi, 0)$ into $\bar{R}$ with the additional conditions that $0 \leqq \phi(x) \leqq u(x, h)$, which can easily be realized.

Let $\pi(x, t)$ be defined as before and let $z(x, t)$ be the solution to the problem.

$$
\begin{array}{rlrl}
\Delta z & =z_{t}, & x \in R_{1} t & >0, \\
z(x, 0) & =u(x, h)-\phi(x), & x \in \bar{R}, \\
z(\xi, t) & =\psi(\xi, t+h)-\psi(\xi, 0) ; & \xi \in B, t \geqq 0,
\end{array}
$$

so that $z(x, t)$ is positive. Clearly then, by the uniqueness of the solution of the Dirichlet problem,

$$
v(x, t)=z(x, t)+\pi(x, t)
$$

and is then positive too. Thus $u(x, t)$ is positive, nondecreasing, and bounded (the boundedness following from the maximum principle). 
Hence $u(x, t)$ approaches a limit $\bar{u}(x)$ as $t$ tends to infinity.

We next show that $\bar{u}(x)$ is harmonic. Let $x^{0}$ be an arbitrary point in $R$, and let $S$ be a sphere center $x^{0}$ and radius equal to one half the distance to $B$. Let $T$ be the boundary of the sphere. And let $G_{S}(x, y, t)$ be the Green's function for the sphere. Then for $t>0, h>0$ we have

$$
\begin{aligned}
u(x, t+h)= & \int_{S} G_{S}(x, y, t) u(y, h) d y \\
& -\int_{0}^{t} \int_{T} \frac{\partial}{\partial n_{y}} G_{S}(x, y, t-\tau) u(y, t-\tau+h) d A_{y} d \tau .
\end{aligned}
$$

Keep $t$ fixed and let $h \rightarrow+\infty$. By the dominated convergence theorem we get

$$
\bar{u}(x)=\int_{S} G_{S}(x, y, t) \bar{u}(y) d y-\int_{0}^{t} \int_{T} \frac{\partial}{\partial n_{y}} G_{S}(x, y, t-\tau) \bar{u}(y) d A_{y} d \tau
$$

which is a solution of the heat equation, and is also independent of $t$, so that it is then a solution of Laplace's equation.

We have only to show that $\bar{u}(x)=u(x)$ where $u(x)$ is the solution to the Dirichlet problem with data $\psi(\xi)$. To see this we note that for each $t>0, \Delta u \geqq 0$, and $u(\xi, t)=\psi(\xi, t) \leqq \psi(\xi)$. Hence $u(x, t)$ belongs to the family of sub-harmonic functions whose supremum gives the solution to the Dirichlet problem. (See Ahlfors [1].) Hence

$$
u(x) \geqq \bar{u}(x) \geqq u(x, t) x \in R, \quad t>0 .
$$

So that as $x \rightarrow \xi$

$\psi(\xi)=\lim u(x) \geqq \lim \sup \bar{u}(x) \geqq \lim \inf \bar{u}(x) \geqq \lim u(x, t)=\psi(\xi, t)$.

Since this is true for all $t$, let $t \rightarrow \infty$ to get

$$
\psi(\xi) \geqq \lim \sup \bar{u}(x) \geqq \lim \inf \bar{u}(x) \geqq \psi(\xi) .
$$

Hence $\lim _{x \rightarrow \xi} \bar{u}(x)=\psi(\xi)$, which implies $\bar{u}(x) \equiv u(x)$.

To complete our proof we invoke a theorem of Dini (see [2]) which states that if a monotone sequence of continuous functions converges to a continuous limit in a closed domain, the convergence must then be uniform.

\section{BIBLIOGRAPHY}

1. L. Ahlfors, Complex analysis, New York, 1953.

2. R. Courant and D. Hilbert, Methods of mathematical physics, vol. 1, New York, 1953.

3. O. D. Kellogg, Foundations of potential theory, New York, 1929. 
4. A. N. Milgram and P. C. Rosenbloom, Harmonic forms and heat conduction I: closed Riemannian manifolds, Proc. Nat. Acad. Sci. U.S.A. vol. 37 (1951).

5. - Heat conduction on Riemannian manifolds II : heat distribution on complexes and approximation theory, Proc. Nat. Acad. Sci. U.S.A. vol. 37 (1951).

6. L. Nirenberg, A strong maximum principle for parabolic equations, Comm. on Pure and Appl. Math. vol. 6 (1953).

7. P. C. Rosenbloom, Notes on partial differential equations, University of Minnesota (in preparation).

8. A. Tychonoff, Sur l'equation de la chaleur de plusieurs variables, Bulletin de l'Université d'Etat de Moscow. I. (1938).

9. D. V. Widder, Positive temperatures on an infinite rod, Trans. Amer. Math. Soc. vol. 55 (1944).

UNIVERSITY OF MINNESOTA

\section{NOTE ON PLURISUBHARMONIC AND HARTOGS FUNCTIONS}

\section{H. J. BREMERMANN}

1. In a recent paper [7] the author has disproved the conjectures that every plurisubharmonic function is a Hartogs function and that every plurisubharmonic function possesses a plurisubharmonic continuation into the envelope of holomorphy of its domain of definition. While the disproof of the first conjecture is rather elementary the disproof of the second conjecture in [7] involves more powerful means.

Now H. Grauert observed in a discussion with the author that by using the same counterexample but passing from tube domains to Reinhardt circular domains the disproof of the continuation conjecture can be conducted in a more elementary way. We carry out this argument here. It furnishes at the same time also a disproof of the first conjecture.

2. The definitions of the Hartogs functions and the plurisubharmonic functions can be found in Bochner-Martin [3], Lelong [9], Hitotumatu [8], and Bremermann [5] and mainly [7]. Every upper semi-continuous Hartogs function is a plurisubharmonic function. Bochner-Martin conjectured that conversely every plurisubharmonic function is a Hartogs function (Bochner-Martin [3, p. 145], compare

Received by the editors July 12, 1955. 